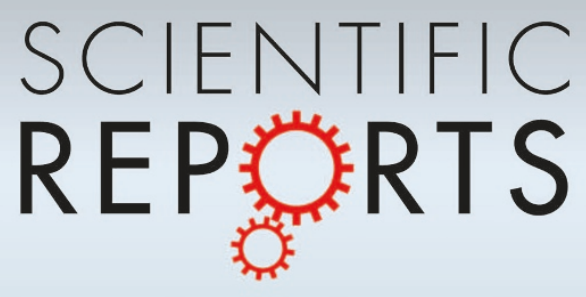

OPEN

SUBJECT AREAS:

LITHOGRAPHY

CHARACTERIZATION AND

ANALYTICAL

TECHNIQUES

PHOTONIC CRYSTALS

POLYMERS

Received

8 April 2013

Accepted

13 August 2013

Published

30 August 2013

Correspondence and requests for materials should be addressed to

T.S. (tina@physik.tuberlin.de)

\section{Transition of refractive index contrast in course of grating growth}

\author{
Tina Sabel' \& Michael Zschocher ${ }^{2}$
}

'Institute for Optics and Atomic Physics, now at Chemical Department, Technische Universität Berlin, Strasse des 17. Juni 135, Berlin, 10623, Germany, ${ }^{2}$ freelance, Pankstrasse 12, Berlin, 13127, Germany.

Studies on the dynamics of holographic pattern formation in photosensitive polymers, gaining deeper insight into the specific material transformations, are essential for improvements in holographic recording as well as in integrated optics. Here we investigate the kinetics of volume hologram formation in an organic cationic ring-opening polymerization system. The time evolution of the grating strength and the grating phase is presented. We found two steps of growth, separated by a depletion of the light diffraction. Capable to explore this growing behavior, a transition-theory of the refractive index contrast is established. Accordingly the growth curves appear to be ruled by the interplay of polymerization and diffusion. Hence the grating formation mechanisms can be qualified as competing effects regarding the contribution to the refractive index change. We investigate the influence of the preparation and exposure procedure on the transition and consider the usability for integrated wave guide functions.

olography and photolithography arise in a powerful combination to create complex structures for micromechanical and photonic devices ${ }^{1,2}$ with potential applications in optics and electronics, as well as for tissue engineering, cell biology and medicinal science ${ }^{3}$. Fundamental material research on pattern formation is prerequisite for future innovations in all those fields ${ }^{4}$. Particularly the dynamics of volume hologram formation in photopolymers is of high interest for practical applications $s^{5}$, mainly with respect to the high flexibility and optimization ability of photopolymers ${ }^{6}$, and thus under extensive studies ${ }^{7-9}$. Appropriate models for the freeradical polymerization are continually improved and extended ${ }^{10-12}$. The performance of cationic ring-opening polymerization (CROP) systems differ in many respects from those of the free-radical polymerization ${ }^{13,14}$. The low termination rate results in living polymerization, with the result of significant dark reaction ${ }^{15}$. Advantages and performance appraisal criteria are no need for solvent processing, good dimensional stability, variable thickness, high energetic sensitivity, large dynamic range and sharp angular selectivity ${ }^{16-19}$. Here we investigate an epoxy based guest-host CROP material, developed by microresist technology, with a convincing performance and a free surface, showing great promise for advanced integrated systems ${ }^{16}$. The results section is structured as follows. Firstly we present the real-time observation of the grating formation. Subsequently we describe how far the found two-step growth infringes the expectations, based on appropriate models. Earlier observations of two-step grating growth notwithstanding ${ }^{20}$, comprehensive investigation and adequate interpretation of this effect remains outstanding. Consequently and specifically for the purpose of interpreting the growing behavior a $\Delta \mathrm{n}$-transitiontheory is established. The third paragraph presents a grating phase shift measurement for corroboration of the theory. The final part deals with consequences regarding the preparation and exposure procedures, i.e. the dependence of the $\Delta \mathrm{n}$-transition on the grating period, on the material's viscosity and on the exposure duration. The corresponding experimental results meet the expectations from the $\Delta \mathrm{n}$-transition theory introduced here.

\section{Results}

Grating growth. The experimental result of the time resolved diffraction efficiency $\eta(t)$ exhibits two separated steps of growth. With the objective of standardization we define the dimensionless time $\tau=\frac{t}{t_{T}}$, where $t_{T}$ is the time separating the two growth steps: $\eta\left(t_{T}\right)=0$ with $t_{T} \neq 0$. A typical growth curve is shown in Fig. 1 . The first growth starts with the beginning of the exposure at $\tau=0$. According to the living polymerization it does not stop at the end of the exposure. The first growth is separated from the second growth by a depletion of the diffraction efficiency around $\tau=1$. For $\tau>1$ the efficiency rises again up to a saturation value. The growth curve in Fig. 1 corresponds to an exposure time of 15 seconds and $t_{T}=200 \mathrm{~s}$.

To explore the observed growth curve progression we analyze the cause of diffraction. The first order diffraction efficiency $\eta$ at a given probe wavelength $\lambda$ and probe angle $\vartheta_{p}$ depends on the layer thickness $d$ and the 


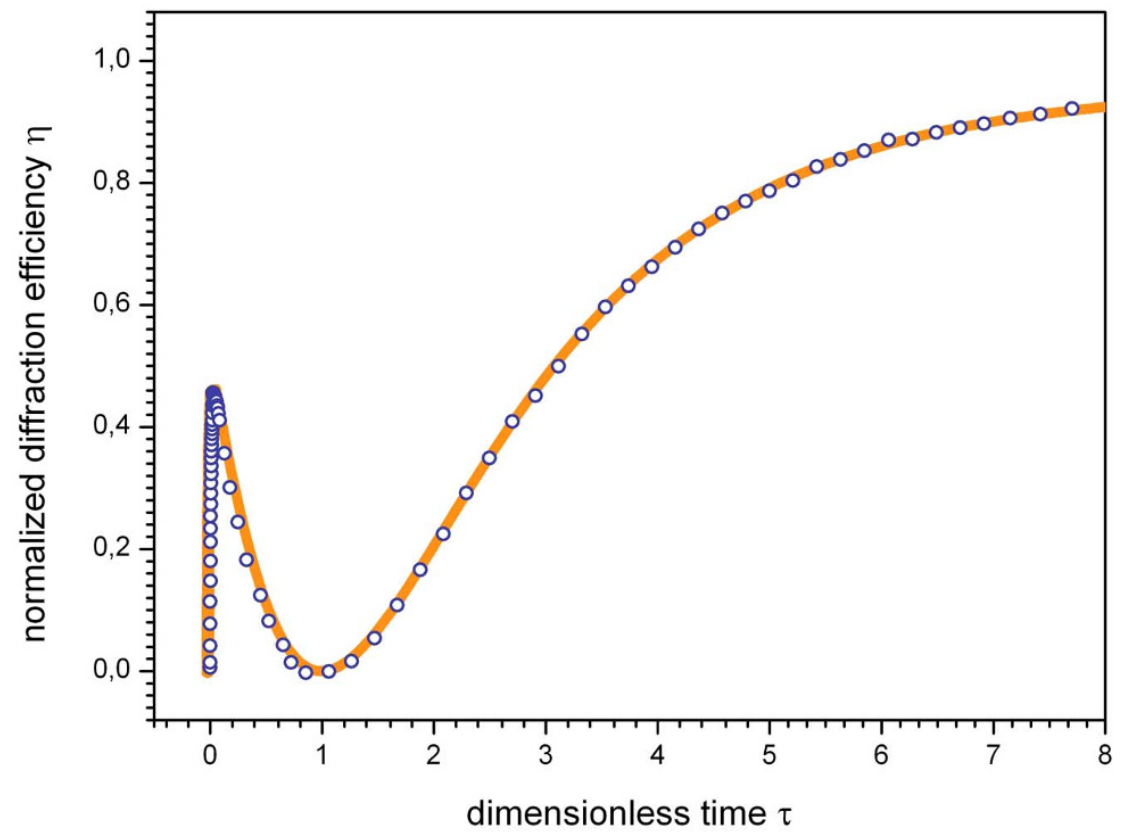

Figure $1 \mid$ Grating growth. The time evolution of the diffraction efficiency shows two separated growth steps: measurement (dots) and calculation (line).

refractive index contrast $\Delta n$. Attention should be paid to the independence on the sign of $\Delta n$ :

$$
\eta=\sin ^{2}(v) \quad v=\frac{\pi d \Delta n}{2 \lambda \cos \left(\vartheta_{p}\right)} .
$$

We maintain probe wavelength, probe angle and layer thickness. Thus the grating growth can clearly be assigned to the time evolution of the refractive index contrast. The most evident potential cause for a decrease of the diffraction efficiency by means of equation (1) is overmodulation $^{21,22}$ as a result of $\Delta n>\lambda \cos \left(\vartheta_{p}\right) / d$. This condition can easily be fulfilled, since we are working with thick layers $(200 \mu \mathrm{m})$ and the inducible refractive index contrast can reach high values (above $\left.5 \cdot 10^{-3}\right)^{16}$. To exclude overmodulation as possible cause for the growing behavior shown here, we create gratings with sufficient low exposure energy density (below $\left.0.3 \mathrm{~J} / \mathrm{cm}^{2}\right)^{16}$, resulting in sufficiently small refractive index contrast (below $\left.2 \cdot 10^{-3}\right)^{16}$.

Modeling. For modeling of the grating growth the time evolution of $\Delta \mathrm{n}$ is needed. Parameter to be taken into account for a change of the refractive index beyond chemical composition are, according to the Clausius-Mossotti relation: molecular polarizability, density and molar mass ${ }^{23}$. In general a saturated growth is expected from theory $^{24}$ and was confirmed experimentally ${ }^{25,26}$. A reasonable assumption $^{27}$ is

$$
\Delta n(t)=s\left[1-e^{-r\left(t-t_{0}\right)}\right]
$$

where $r$ controls the rise, $s=\Delta n(t \rightarrow \infty)$ is the saturation value and $t_{0}$ the starting time of the grating growth. At any rate the growth curves we observed are not in compliance with this condition. This necessitates model extension and additional assumptions.

To explore the growth curve progression shown in Fig. 1 we hypothesize a transition of the refractive index contrast at $\tau=1$. As a consequence of the square sine symmetry of equation (1) the diffraction efficiency is independent on the sign of $\Delta \mathrm{n}$. Whereas a $\Delta \mathrm{n}$ change of sign must cause a zero point of diffraction. We call this zero point the transition point, starting from the assumption that the system develops initially a positive contribution to the refractive index change $\left(\partial_{n}>0 \Rightarrow \Delta n>0\right)$, which is finally predominated by a negative change $\left(\partial_{n}<0\right)$, with the result of $\Delta n<0$ for $\tau>1$. The appropriate mechanisms are in line with the expectations, based on the system design and will be described below. An overview of the based physico-chemical processes and schematic diagram of the refractive index change according to the $\Delta \mathrm{n}$-transition is displayed in Fig. 2.

The first step of growth, we reckon to be attributed to a positive refractive index contrast, is presumably caused by ring opening and covalent bond on the host system. This is plausible under the conception, that charge transfer on the molecular level is more easily induced in case of an open ring, considering the localization of the charge distant from the nuclei. The arithmetical reasons can be found in the molar refraction model. According to the correlation with the chemical structure the refractive index can be calculated using the atomic-, group and bond contributions to the molar refraction. Herein the ring structure represents an constitutional increment with a negative $\operatorname{sign}^{23}$. Consequently the opening of the ring results in a positive contribution to the molar refraction. Hence already the ring-opening of the functional group, which is the initial step of the grating formation, prior to cross linking and a fortiori prior to diffusion could solely induce a positive change in the refractive index.

The second growth step most likely correlates to component segregation throughout diffusion. The guest with regard to a low molecular weigh serves as diffusing component. The guest component is low refractive index species (LRIS), compared to the host system $^{16}$. As a consequence monomer diffusion results in a negative contribution to the refractive index change (see Fig. 2, line 4). However, the effect of densification in the course of monomer diffusion may inhibit the second growth step by means of a contribution which, in turn, increases the refractive index. Further investigations on time- and spatially resolved Bragg-angle detuning, providing information on volume deformations, could contribute to entirely explain the grating formation mechanism, including effects of material shrinkage or expansion during diffusion or counterdiffusion of components.

Under the assumptions of the $\Delta$ n-transition the amplification of the model consists in an additive composition of positive and negative index contrast, each according to equation (2). Thereby the parameter range expands to $\left\{r, s, t_{0}\right\}_{+,-}$, with respect to the positive and the negative $\Delta \mathrm{n}$-contribution, respectively. The fit-function, presented in Fig. 1 is calculated based on this amplified model. The 

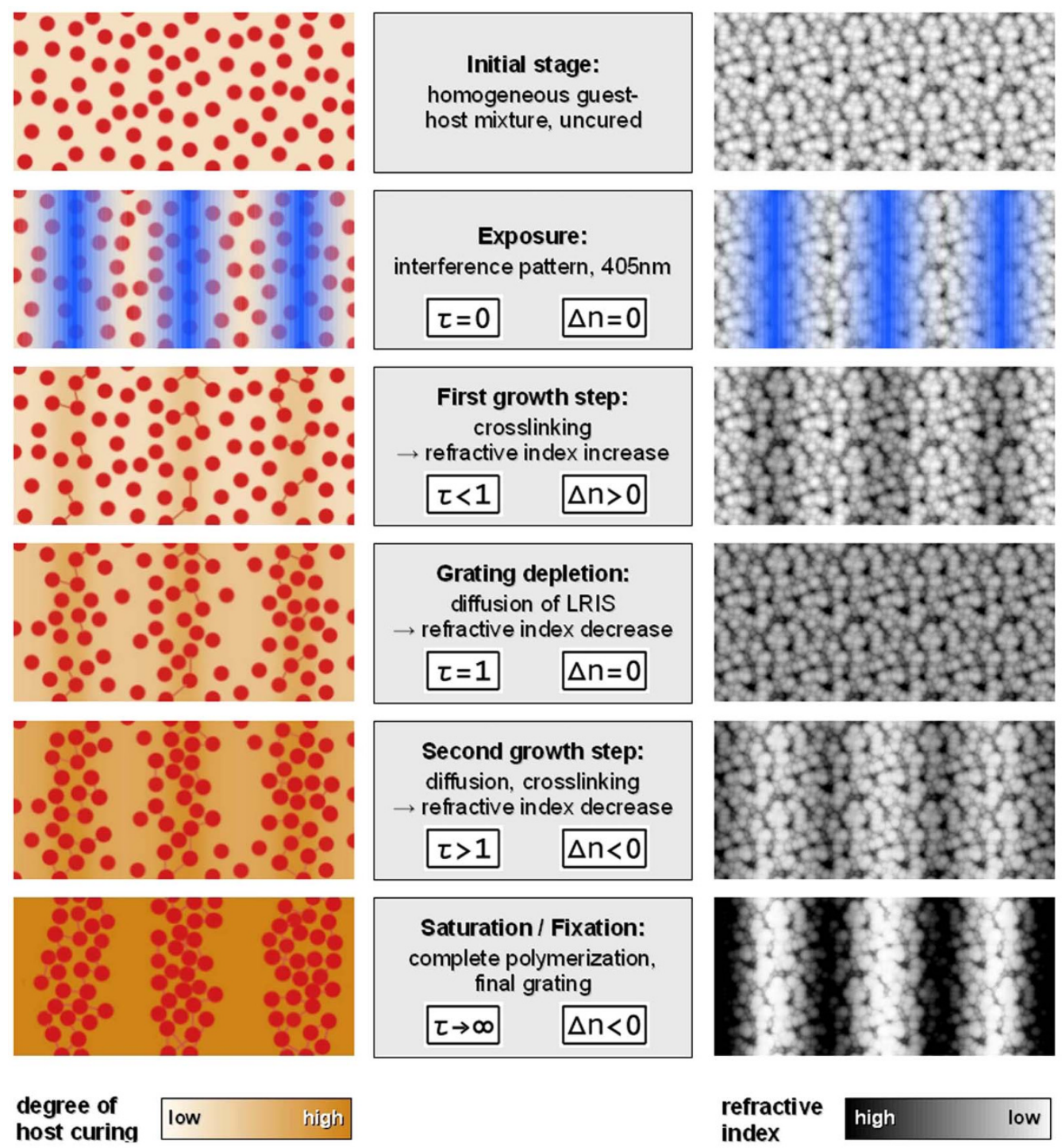

Figure $2 \mid$ Schematic diagram of the $\Delta \mathrm{n}$-transition theory: Impact of the polymerization-diffusion interaction (left column) on the refractive index modulation (right column). The chemically and optically homogeneous uncured mixture (first line) is holographically exposed at $\tau=0$ (line 2 ). Photosensitive compounds initiate crosslinking of host and guest components (background in shades of ochre and red dots, respectively). The initial crosslinking causes a local refractive index increase, resulting in a positive index contrast for $\tau<1$ (line 3). Polymerization initiates guest component diffusion, subsequently segregating low refractive index species (LRIS) (line 4, left column). The involved refractive index decrease depletes the refractive index contrast and erases the grating (line 4, right column). At this moment (transition point, $\tau=1$ ) the chemically inhomogeneous mixture features optically homogeneous properties. Proceeding diffusion with the associated refractive index decrease creates a grating with negative index contrast at $\tau>$ 1 (line 5). Subsequently the final saturated grating is formed by means of complete polymerization (line 6).

corresponding fit parameter are $r_{+}=65, r_{-}=0.12, s_{+}=1 \cdot 10^{-3}, s_{-}$ $=-2.7 \cdot 10^{-3}$ and $t_{0+}=t_{0-}=0$.

Grating phase shift. To find evidence for the hypothesis of the $\Delta \mathrm{n}$ transition we implemented a grating phase shift measurement. Suzuki et al. apply phase-shift measurements to identify particle diffusion or counterdiffusion during hologram formation ${ }^{28}$. In our case a change of sign in the refractive index contrast should be accompanied by a grating phase shift of 180 degree (see Fig. 2, right column: transition from line 3-5). For that purpose we observed the interference of the first diffraction order with the transmitted light during the grating formation process.

Figure 3 shows the grating phase and the corresponding growth curve on a logarithmic dimensionless time scale. The growth curve in Fig. 3 corresponds to an exposure time of 15 seconds and $t_{T}=7500 \mathrm{~s}$. The phase shift is clearly visible from Fig. 3 , as well as the coincidence with the depletion time $t_{T}$. Theoretically it is expected to find the grating phase shift sharply located at the transition point $(\tau=1)$. As apparent from Fig. 3 deviations are caused under real conditions. The grating phase shift in Fig. 3 is located between $\tau=0.5$ and $\tau=2$. The fact that the grating phase shift spreads over a period around $\tau=1$ appears plausible under the assumption that the transition does not happen simultaneously all over the probe beam diameter, according to a dependence on the recording intensity. The grating phase shift measurement corroborates the $\Delta \mathrm{n}$-transition theory: an initial, light induced, positive change of the refractive index is gradually converted to a negative one, triggered by diffusion of low index species. While the phase shift of 180 degree shows the inversion of the lattice planes (see Fig. 2, line 3-5).

Scaling and incomplete null. Having set up and corroborated the theory of a $\Delta$ n-transition, we prospect for possible consequences regarding the preparation and exposure procedures. Caused by sufficient low recording intensity the so called diffusion-free 

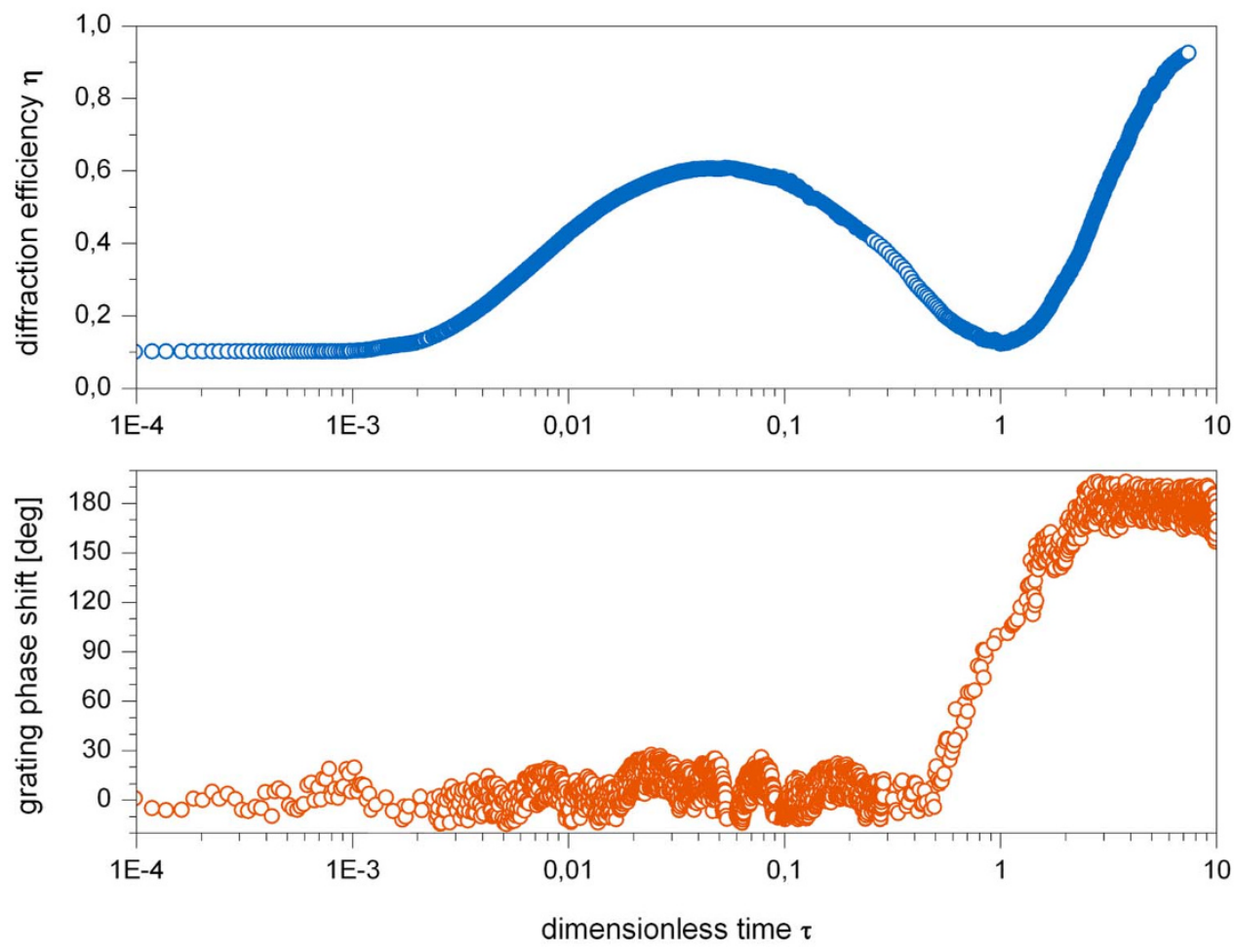

Figure $3 \mid$ Grating phase shift. The time evolution of the grating phase during grating growth corroborates a transition of the refractive index contrast between the first and the second step of growth.

condition $^{29}$ is attended by a high $\mathrm{R}$ parameter. According to the definition

$$
R=|K|^{2} \frac{D}{F}
$$

with $K$ the grating vector, $D$ the diffusion coefficient and $F$ half the maximum polymerization rate, this corresponds to slow polymerization, fast diffusion and/or small grating periods. Thus a dependence of the $\Delta \mathrm{n}$-transition process, particularly of the transition time on the preparation and exposure procedure is expected. First of all the grating vector $K$ can directly be adjusted by means of the exposure geometry. Secondly the diffusion coefficient $D$ is influenced by the material's viscosity, which is aligned through sample preparation ${ }^{16}$. Finally the polymerization rate $F$ depends on the amount of exposure and therewith on the exposure duration. All in all the $\mathrm{R}$ parameter and therewith the grating growth can be controlled by means of internal and external influencing factors accessible via preparation and exposure procedures.

Firstly the time period required for the transition is supposed to depend on the grating period. Increasing grating periods should result, with respect to an enlargement of the diffusion length, in an increase of the transition time. Experimental confirmation is given in terms of characteristic growth curves in reference to different grating periods.

Figure 4 shows growth curves on logarithmic time scale with grating periods of $140 \mathrm{~nm}, 2 \mu \mathrm{m}, 3 \mu \mathrm{m}$ and $7 \mu \mathrm{m}$ respectively, whereas exposure time was 5 seconds in case of the $2 \mu \mathrm{m}$-grating and 15 seconds in all other cases. Very small grating periods, i.e. a high $\mathrm{R}$ parameter (Fig. 4 top left) results in a single growth step. The refractive index contrast is presumably negative from the very start of grating growth, with no transition taking place, due to the very short diffusion length. Diametrically opposed to this very large grating periods, i.e. a low $\mathrm{R}$ parameter corresponds to an exceedingly pronounced transition (Fig. 4 bottom right). A stationary balance of positive and negative change of the refractive index seems to last for several minutes before the depletion slowly starts. In between those extreme cases the intermediate behavior constitutes the characteristic two-step growth. Thereby shorter grating periods, lower viscosity and/or short exposure duration, i.e. a high $\mathrm{R}$ parameter results in shorter transition time. At the threshold (Fig. 4 top right) the first step of growth is only visible on a double logarithmic scale. A decrease of the $\mathrm{R}$ parameter by means of increasing grating periods, longer exposure duration and/or higher viscosity results in prolonged transition (Fig. 4 bottom left), i.e. increasing transition time. In particular the corresponding transition times for the intermediate growth curves (Fig. 4 top right and bottom left) are $85 \mathrm{~s}$ and $4000 \mathrm{~s}$, respectively.

More importantly the material's viscosity should have an impact on the scaling of the $\Delta \mathrm{n}$-transition by influencing the diffusion coefficient $D$, what is to be thoroughly investigated. After spincoating the polymer samples are dried on a hotplate. The viscosity depends on the duration of this prebake procedure. Figure 5 shows growth curves on a double logarithmic scale, with samples differing in prebake duration. On the real time scale the extension of the transition time with increasing prebake duration is clearly distinguishable. Thus the experimental results meet the expectations from the $\Delta \mathrm{n}$-transition theory. Prebake duration was 90, 120 and 180 minutes and transition times are 80, 160 and 600 seconds, respectively.

Another conspicuous feature, readily identifiable from Fig. 5 is an increasingly pronounced incomplete null diffraction at the transition time $t_{T}$. The depletion of light diffraction is less pronounced, the stronger the diffusion process is assumedly inhibited by higher viscosity. In such a case the Gaussian profile of the exposure beam, thus the intensity-dependence of the transition time, seems no longer negligible. The in-situ measurement of the diffraction efficiency is always average determination over the probe beam diameter. If the intensity-dependence of the polymerization and/or diffusion rate over this diameter results in significantly unequal transition times the average value of the diffraction efficiency is non-zero, with the result of an incomplete null in the growth curve. 


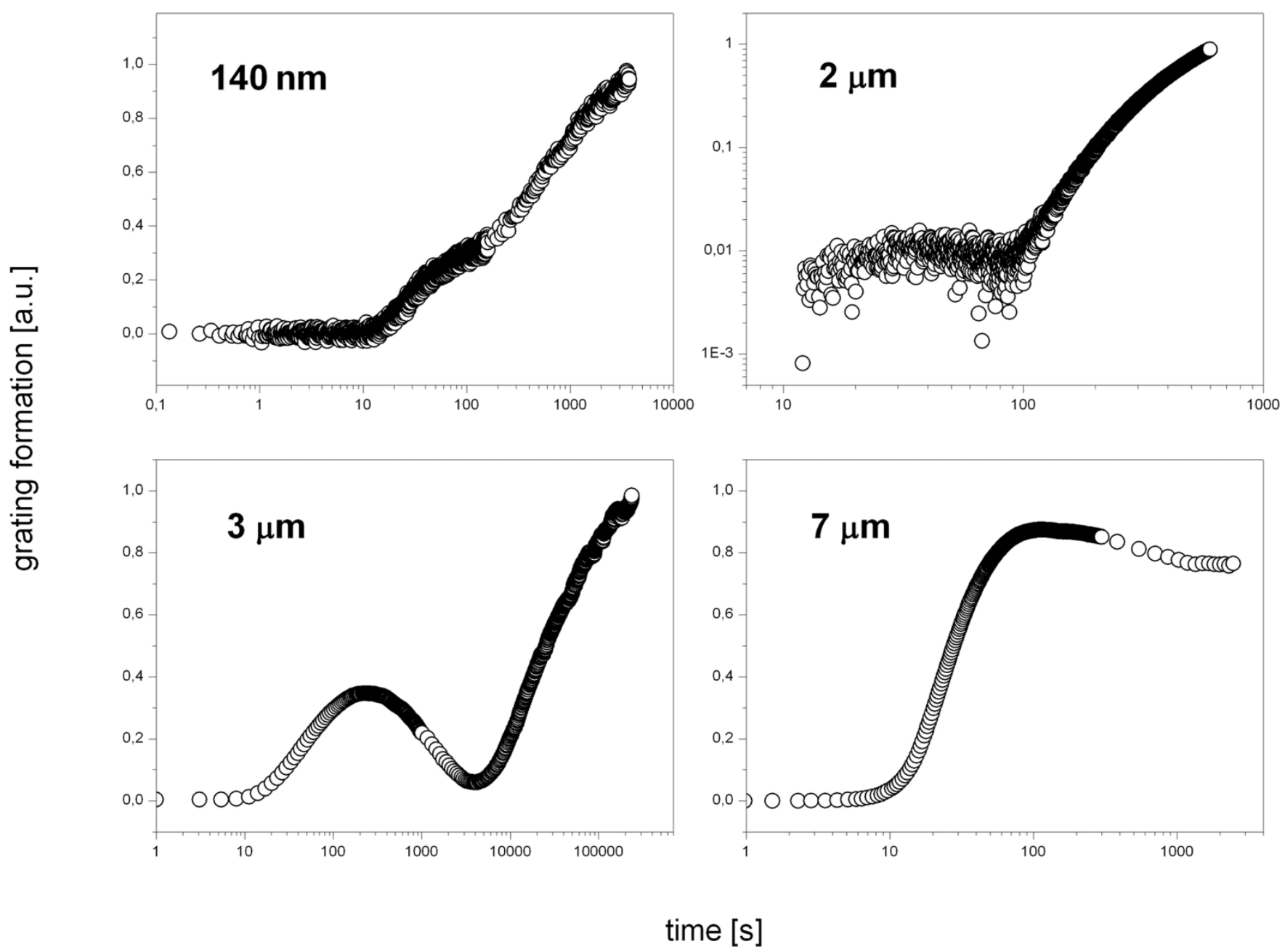

Figure $4 \mid$ Influence of the grating period. Growth curves for various lattice spacings highlight the impact of the exposure procedure.

In this context the data presented in Fig. 6 supports the $\Delta$ n-transition-theory. It displays the dependence of the transition time and the characteristic of the incomplete null on exposure and prebake duration.

The exposure duration controls the scaling of the transition process. The longer the exposure the later the transition takes place, which can be traced to the increase of the polymerization rate $F$. Stronger initiation of polymerization requires more diffusion to trigger the transition. The markedness of the correlation depends on the materials viscosity, which is aligned by prebake and in turn determines the diffusion coefficient $D$. Figure 6 shows that the transition time and the magnitude of the incomplete null is increasing with increasing prebake duration. This is in compliance with the implications from the transition theory, as stated above.

It is also identifiable from Fig. 6 that in case of short prebake duration the dependence on exposure duration is weak. While with increasing prebake duration the dependence on exposure duration is increasing. This supports the exposed assumptions on the intensitydependence of the transition process.

\section{Discussion}

In conclusion we reveal potential impact of living polymerization. In course of the grating formation process a positive change of the refractive index contrast on a molecular level and the negative contribution due to component segregation seem to act as competing effects, taking place on overlapping time scales with the result of characteristic two-step growth curves. The indicator to be observed

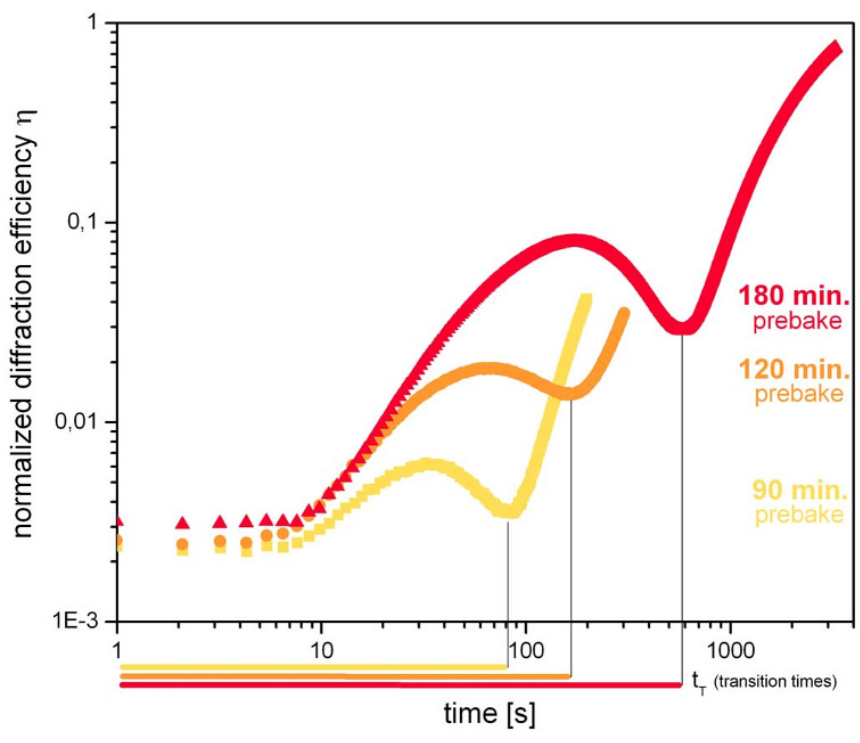

Figure $5 \mid$ Viscosity effects. The grating growth strongly depends on the prebake duration. 

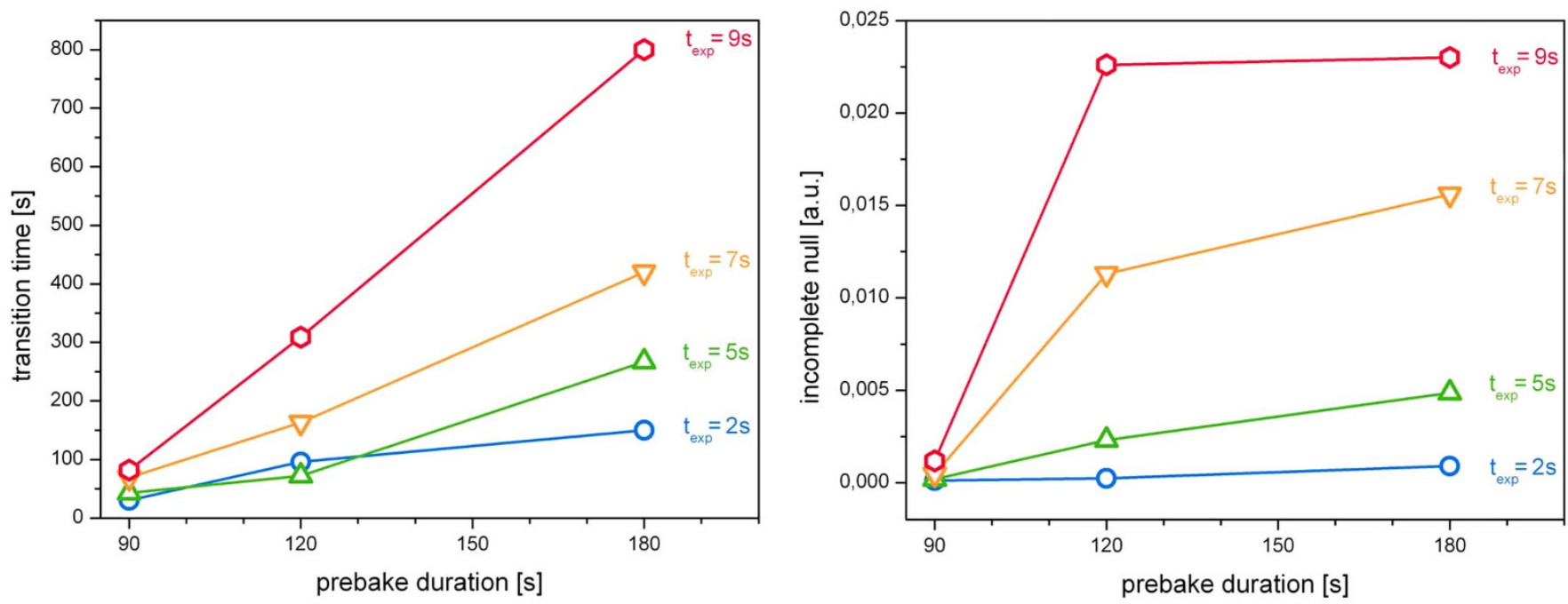

Figure 6 Scaling and incomplete null. The scaling of the transition time and pronounce of the incomplete null in arbitrary units is displayed, depending on prebake duration and exposure duration, respectively.

optically is a function of the absolute value of a material property. Thus it provides information only on the amount of the causal processes, not on their individual directions. Whereas the sign of the refractive index contrast is irrelevant for diffractive issues, it becomes particularly important with regard to integrated optics. The intrinsically negative refractive index contrast notwithstanding, wave guide functions are still feasible. In fact such a local positive change, caused by inhibited diffusion, can be realized in terms of nonsinusoidal refractive index profiles ${ }^{16}$ and might be amplified utilizing the transition effects described here. The dependence of the grating growth on internal and external influencing factors is described. Altogether the presented results provide instruments to control hologram formation and demonstrate the potential of optical techniques as analytical methods. We compile the theory of a $\Delta$ n-transition, assumedly triggered by a predominance of low index species diffusion, accommodating experimental results and system design.

\section{Methods}

Our investigations are based on the recording and analysis of one-dimensional, plane-wave volume holograms of both transmission- and reflection type. The holographic exposure setup features two freely propagating recording beams $(405 \mathrm{~nm})$ plus an in-situ part for real-time observation of the grating formation. Details on sample preparation and hologram recording are given in Ref. 16. The in-situ part consists of a first order Bragg-matched $633 \mathrm{~nm}$ HeNe laser and a position sensitive device (PSD) as photodetector.

1. Lourtioz, J.-M. Photonic crystals: Writing 3D photonic structures with light. Nature Mat. 3, 427-428 (2004).

2. Campbell, M., Sharp, D. N., Harrison, M. T., Denning, R. G. \& Turberfield, A. J. Fabrication of photonic crystals for the visible spectrum by holographic lithography. Nature 404, 53-56 (2000).

3. Nie, Z. \& Kumacheva, E. Patterning surfaces with functional polymers. Nature Mat. 7, 277-290 (2008).

4. Erdmann, A., Shao, F., Agudelo, V., Fühner, T. \& Evanschitzky, P. Modeling of mask diffraction and projection imaging for advanced optical and EUV lithography. J. Mod. Opt. 58, 480-495 (2011).

5. Neipp, C. et al. Angular responses of the first and second diffracted orders in transmission diffraction grating recorded on photopolymer material. Opt. Express 11, 1835-1843 (2003)

6. Guo, J., Gleeson, M. R. \& Sheridan, J. T. A Review of the Optimisation of Photopolymer Materials for Holographic Data Storage. Phys. Res. Int. 2012, 803439 (2012)

7. Trout, T. J., Schmieg, J. J., Gambogi, W. J. \& Weber, A. M. Optical Photopolymers: Design and Applications. Adv. Mater. 10, 1219-1224 (1998).

8. Colvin, V. L., Larson, R. G., Harris, A. L. \& Schilling, M. L. Quantitative model of volume hologram formation in photopolymers. J. Appl. Phys. 81, 5913-5923 (1997).

9. Waldmann, D. A. et al. Cationic ring-opening photopolymerization methods for volume hologram recording. Proc. SPIE 2689, 127-141 (1996).
10. Gleeson, M. R., Guo, J. \& Sheridan, J. T. Optimisation of photopolymers for holographic applications using the Non-local Photo-polymerization Driven Diffusion model. Opt. Express 19, 22423-22436 (2011).

11. Kelly, J. V., Gleeson, M. R., Close, C. E., O’Neill, F. T. \& Sheridan, J. T. Temporal response and first order volume changes during grating formation in photopolymers. J. Appl. Phys. 99, 113105 (2006).

12. Babeva, T., Naydenova, I., Mackey, D., Martin, S. \& Toal, V. Two-way diffusion model for short-exposure holographic grating formation in acrylamide-based photopolymer. J. Opt. Soc. Am. B 27, 197-203 (2010).

13. Wei, H.-Y. et al. Holographic grating formation in cationic photopolymers with dark reaction. Chin. Phys. Lett. 23, 2960-2963 (2006).

14. Ingwall, R. T. \& Waldman, D. A. CROP photopolymers for volume hologram recording. SPIE International Technical Group Newsletter 11, 1, 7 (2000).

15. Decker, C. \& Moussa, K. Kinetic study of the cationic photopolymerization of epoxy monomers. J. Polym. Sci. A Polym. Chem. 28, 3429-3443 (1990).

16. Sabel, T., Orlic, S., Pfeiffer, K., Ostrzinski, U. \& Grützner, G. Free-surface photopolymerizable recording material for volume holography. Opt. Mat. Express 3, 329-338 (2013).

17. Ingwall, R. T. \& Waldmann, D. Photopolymer Systems, in Holographic Data Storage, Coufal, H. J., Psaltis, D., Sincerbox, G. T. (Eds.) (Springer, 2000).

18. Jeong, Y.-C., Lee, S. \& Park, J.-K. Holographic diffraction gratings with enhanced sensitivity based on epoxy-resin photopolymers. Opt. Express 15, 1497-1504 (2007).

19. Schilling, M. L. et al. Acrylate Oligomer-based photopolymers for optical storage applications. Chem. Mater. 11, 247-254 (1999).

20. Colburn, W. S. \& Haines, K. A. Volume Hologram Formation in Photopolymer Materials. Appl. Opt. 10, 1636-1641 (1971).

21. Sakhno, O. V., Goldenberg, L. M., Smirnova, T. N. \& Stumpe, J. Holographic patterning of organic-inorganic photopolymerizable nanocomposites. Proc. SPIE 7487 (2009).

22. Tomita, Y. Holographic assembly of nanoparticles in photopolymers for photonic applications. SPIE 10.1117/2.1200612.0475 (2006).

23. van Krevelen, D. W. \& te Nijenhuis, K. Properties of Polymers. (Elsevier, 2009).

24. Kelly, J. V. et al. Temporal analysis of grating formation in photopolymer using the nonlocal polymerization-driven diffusion model. Opt. Express 13, 6990-7004 (2005).

25. Cho, Y. H., Shin, C. W., Kim, N., Kim, B. K. \& Kawakami, Y. High-Performance Transmission Holographic Gratings via Different Polymerization Rates of Dipentaerythritol Acrylates and Siloxane-Containing Epoxides. Chem. Mater. 17, 6263-6271 (2005).

26. del Monte, F., Martinez, O., Rodrigo, J. A., Calvo, M. L. \& Cheben, P. A volume holographic sol-gel material with large enhancement of dynamic range by incorporation of high refractive index species. Adv. Mat. 18, 2014-2017 (2006).

27. Gallego, S. et al. Direct analysis of monomer diffusion times in polyvinyl/ acrylamide materials. Appl. Phys. Lett. 92, 073306 (2008).

28. Suzuki, N. \& Tomita, Y. Real-time phase-shift measurement during formation of a volume holographic grating in nanocomposite-dispersed photopolymers. Appl. Phys. Lett. 88, 011105 (2006).

29. Piazzolla, S. \& Jenkins, B. K. Holographic grating formation in photopolymers. Opt. Lett. 21, 1075-1077 (1996)

\section{Acknowledgements}

We acknowledge support by the co:bios foundation. We thank Dr. Karl Pfeiffer and microresist technology $\mathrm{GmbH}$ for developing and providing the photopolymer material 
and samples. We thank Andreas Erdmann from Fraunhofer IISB and Alexandre Tishchenko from Laboratoire Hubert Curien for reading the manuscript and for valuable indications.

\section{Author contributions}

T.S. performed hologram recording and analysis, analyzed the data and wrote the manuscript. M.Z. was involved in study design and results interpretation and designed the figures. All authors discussed the results and commented on the manuscript.

\section{Additional information}

Competing financial interests: The authors declare no competing financial interests.

How to cite this article: Sabel, T. \& Zschocher, M. Transition of refractive index contrast in course of grating growth. Sci. Rep. 3, 2552; DOI:10.1038/srep02552 (2013).

(c) (i) $($ This work is licensed under a Creative Commons Attribution-

BY NC No NonCommercial-NoDerivs 3.0 Unported license. To view a copy of this license, visit http://creativecommons.org/licenses/by-nc-nd/3.0 